\title{
Software Agents Situated in Primary Distribution Networks: A Cooperative System for Fault and Power Restoration Management
}

\author{
Ioannis S. Baxevanos and Dimitris P. Labridis, Senior Member, IEEE
}

\begin{abstract}
In this paper, extended research upon the potentials of implementing distributed artificial intelligence technology to achieve high degrees of independency in distribution network protection and restoration processes is presented. The work that has already been done in the area of agent-based and/or knowledge-based applications and expert systems is briefly reviewed. The authors justify the need to distribute activities in contradiction to the centralized methodologies. A proper model of the real environment is introduced in order to define the designing parameters of a prototype agent entity, which is the part of a cooperative network-management system. The system's goal is to autonomously perform effective fault management upon medium-voltage power distribution lines. The structure of the agent entity is then described by means of the agent behaviors being implemented. The cooperative operations of the proposed system and its computer simulation are presented. Simulation results are being evaluated. Finally, general conclusive remarks are made.
\end{abstract}

Index Terms-Artificial intelligence, cooperative systems, power distribution protection, underground power distribution lines.

\section{INTRODUCTION}

$\mathbf{T}$ HE generic problem of fault management and power restoration in the areas of power generation, transmission, and especially power distribution networks has proven to be a significant field of research and applications for artificial intelligence. Heuristic algorithms for network reconfiguration in large distribution systems have been proposed by power engineers and researchers [1], [2]. A combination of heuristics and fuzzy logic for estimating the power restoration time in distribution networks, as a part of a generalized optimization method for solving the restoration problem, is presented in [3]. The effectiveness of the joint usage of expert systems and mathematical programming in power systems restoration is presented in [4]. In [5], a restoration aid system for primary distribution substations, based on the combination of expert systems and heuristic rules, is proposed. In addition, several rule-based expert systems, utilizing domain knowledge and reasoning in order to formulate logical problem solutions as

Manuscript received April 11, 2006; revised November 2, 2006. This work was supported in part by the Operational Program for Education and in part by the Initial Vocational Training (O.P. "Education")-Heracletus. Paper no. TPWRD-00199-2006.

The authors are with the Power Systems Laboratory, Department of Electrical and Computer Engineering, Aristotle University of Thessaloniki, Thessaloniki GR 54124, Greece (e-mail: labridis@auth.gr).

Digital Object Identifier 10.1109/TPWRD.2007.905463 auxiliary restoration procedures, have been proposed in [6] and [7].

The evolution of distributed artificial intelligence (DAI) and cooperative systems technology provided techniques and methodologies for the development of management architectures, which exhibit decentralized functionality. Several multiagent systems for both transmission and distribution power networks restoration have been developed [8], [9]. Multiagent systems technology has also been implemented for fault diagnosis in power distribution networks [10] as well as for managing technically advanced medium-voltage (MV) distribution systems [11].

Several distribution automation companies have also developed and produced complete automation systems with distributed characteristics, which may be integrated with distribution supervisory control and data-acquistion (SCADA) architectures or work as stand-alone applications [12].

The objectives of the distributed intelligence approach to distribution network management are the same as the ones for centralized systems: to minimize the duration of outages as well as the customer interruptions, in favor of the overall distribution system reliability. Customer-based distribution reliability indices, especially customer average interruption duration index (CAIDI) and system average interruption duration index (SAIDI) [13], will be potentially improved. However, DAI technology, if implemented, may actually result in self-managing and "self-healing" electrical networks [14].

\section{DistRIBUTING INTELLIGENCE}

The evolution of distribution automation systems with decentralized resources, such as utility SCADA systems, has already provided powerful applications which are able to manage several network topologies, in a quite effective manner. Thus, rational questions rise concerning the implementation of distributed intelligence for the electrical network management. The answers to those questions lay within the following parameters:

- the level up to which utilities need to distribute their systems;

- the benefits of a highly distributed solution in contrast with the centralized systems;

- the actual need of a distributed approach to the networkmanagement problem.

As power distribution systems become more complex, with a lot of interconnections and many potential power sources for the augmenting loads, the functional requirements of current 
automation systems stress their operability. A highly complicated system that meets the above requirements will demand large computational resources, specialized and technically advanced communication networks as well as high expertise of its users. Moreover, cost and constructive constraints impel a relative low distribution of the system interaction with specific electrical network nodes. Actually, current automation systems are implemented in accordance with a network segmentation which is derived from the control engineers' experience on the criticality of loads and, as a result, the criticality of certain network segments. The effectiveness of the network-management systems depends on the areas covered by the segments and the control capabilities over those areas; the smaller the segments can be, the higher the functional accuracy and operational performance of the distribution automation systems is.

On the other hand, the power distribution grid is a physically and highly distributed system depending on the proper functionality and interoperability of several heterogeneous components. In addition, the various grid configurations, which may be derived from restoration procedures, continuously alter the topologies an automation system is initially set for. As a result, common tactics apply additional "returning to normal topology" processes in order for the automation systems to operate in effective manners. Time delays and undesirable customer interferences, raising the system functional costs, are introduced. Therefore, the need for network-management systems, which exhibit self-organizing and self-coordinating behaviors, is imperative.

\section{PROBLEM DESCRIPTION}

The problem that has to be encountered by a distributed intelligent system is formulated as follows.

Premises.

1) Consider an underground multiple primary loop threephase distribution system (Fig. 1), and an MV line that belongs to the above system, considered as the "Main Line" (Fig. 1, LINE 1).

2) Consider a permanent line fault of any type somewhere inside the "Main Line."

3) Finally, consider that the primary distribution substation breaker (Fig. 1, BREAKER 1) has tripped to clear the fault.

Objective.

Design a distributed-management system which will isolate the fault to the "absolutely minimum segment" of the main line and restore power to the "absolutely maximum segment" of the main line, in relation to the loading conditions of the alternative power sources, in the minimum time interval.

The "absolutely minimum segment" is defined as the part of the main line which includes the fault's position and which is bounded by the secondary distribution substations that are adjacent to the fault. The "absolutely maximum segment" is defined as the largest possible part of the main line that can be restored in accordance with the grid topology, the position of the alternative power supplies with respect to the fault as well as the excessive loading capabilities of the latter.

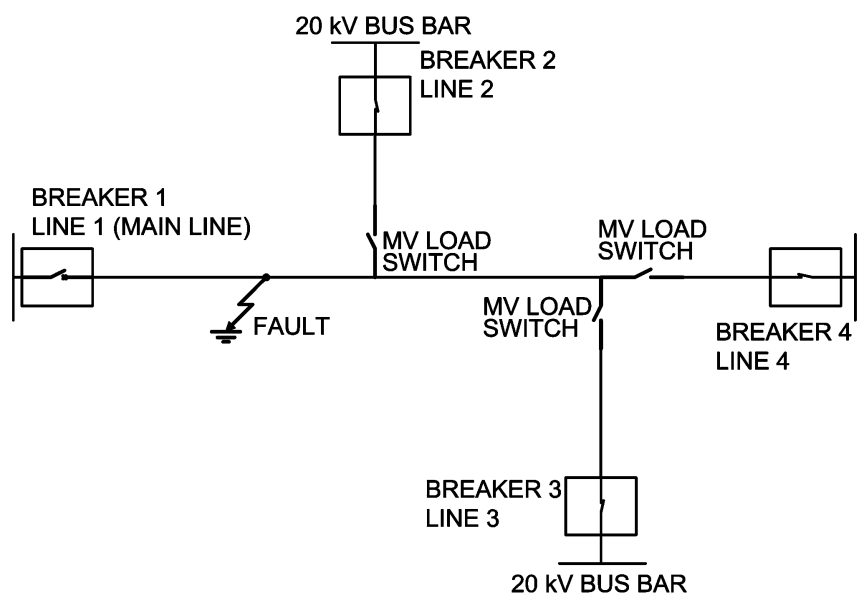

Fig. 1. Problem topology.

Additional constraints to the proposed system design are impelled: The switching operations which take place during the fault isolation and power restoration procedures must follow a certain order so that once the damage has been repaired, the secondary substations that were not restored, will be easily and rapidly reconnected to the main line, without the need for complicated switching operations.

Fig. 1 exemplifies the problem topology. There are not any constraints neither for the number of MV lines that form the distribution system, nor for the number of the secondary substations for each MV line, as long as the open-loop structure is maintained. LINE 1 is the MV distribution line which is considered as the "Main Line." LINE 2, LINE 3, and LINE 4 are the alternative feeders for the loads of the "Main Line." The MV load switches, which are computer controlled, belong to the respective secondary substations which are called "Junction Nodes." A "Junction Node" is a substation located at a grid junction point.

Finally, it should be noted that the switching operations mentioned before concern the MV load switches of the secondary substations which form the open-loop system design.

\section{ENVIRONMENTS}

According to the above problem description, the proposed cooperative system must consist of purely situated agent entities [16] by means of software agents that own an explicit representation of the hosting environment. By making use of that representation, the agents are capable of interacting with the environment, in terms of perceiving the environment state, planning future actions and decisions, and acting upon it.

\section{A. Environment Specification}

The constraints of "absolutely minimum" and "absolutely maximum" segmentation in fault isolation and power restoration procedures, respectively, produce local environments wherein the agents will live and act. In this paper, both secondary and primary distribution substations are designated as the local environments. Therefore, the system consists of primary substation agents (PSAs) and secondary substation agents (SSAs). 
Due to the collaborative nature of the proposed system, an agent's environment may be considered as a synthesis of two environment aspects-physical and relational. An agent entity perceives the physical aspect as operational states of the local substation, such as values of electrical parameters, states of the load switches, etc. The relational aspect concerns the interactions with other agent entities which coexist in local and/or neighboring substations. According to this generalization, agents will cope with nondeterministic and partially inaccessible environments if the metering devices fail to provide all required information due to hardware malfunctions as well as if the interactions with other agents are unattainable due to communication problems. On the other hand, regarding the final actions for managing line faults, the agents' behavior is deterministic: the isolation of an identified fault and the restoration of power to customers require certain and absolutely defined switching actions.

\section{B. Environment Representation}

The logical representation of local environments is structured according to the above specifications. In the agents' development framework, which has been used to develop the situated agents [17], the environment representation was achieved by implementing the framework's ontology [18] support.

As a result, the ontology structure is formulated by representing the following objects for each agent.

- Concepts: agent, substation, switch, breaker, power, and fault position.

- Predicates: owns, sees, neighbors.

The relational aspect of local environments is represented as the type and characteristics of the messages that the agents exchange. In this work, the implementation of agent communication language (ACL) messages [19] of INFORM performative, was selected.

Additional predicates are used in order to enable agents to internally represent the ACL messages content: breaker information, fault detection, breaker action, restoration request, restoration reply, and confirmation.

Concepts and predicates of the system's ontology are programming code classes which provide the proper methods for managing the respective objects' properties. A description of the ontology is included in Appendix A.

\section{Agent Behaviors}

The operation of a system agent depends on the structure of the behaviors that the agent incorporates and upon which the agent has absolute control.

1) SSA Behavior: The general behavior of an SSA is structured according to the system's operational states: steady state, fault localization and isolation state, and power restoration state. The above are internally represented in an SSA as states of a finite state machine behavior. The transitions between states are designated by the agent's reasoning, and the decisions are taken according to environment perceptions.

Steady state. It is a behavior consisting of two subbehaviors.

1) Breaker information receiver-sender. Refers to the functions of receiving and forwarding breaker information messages. For each corresponding message, the agent manages

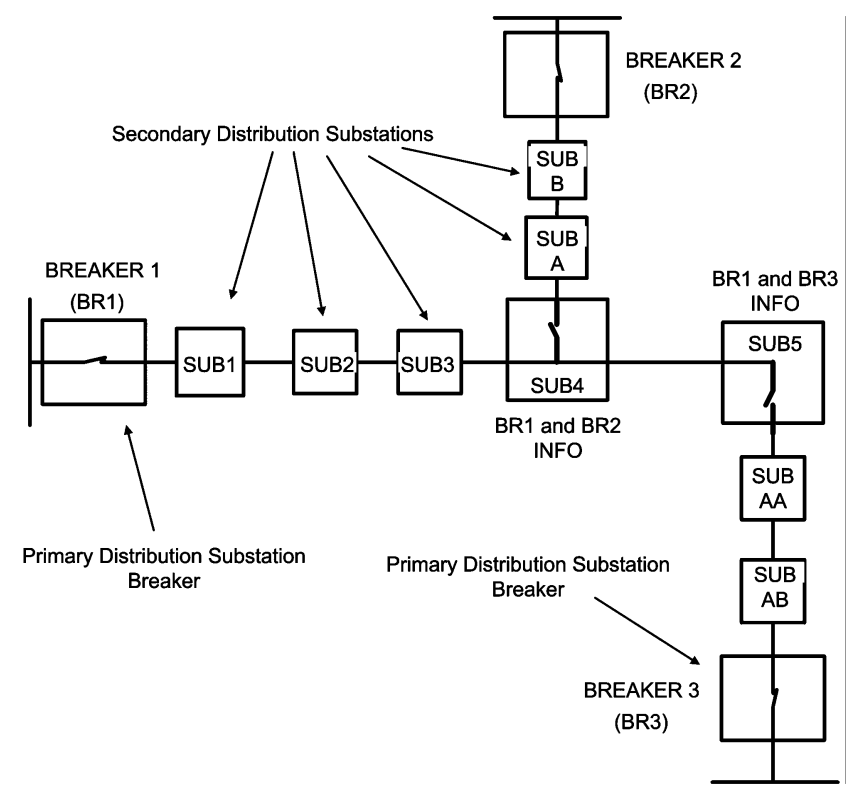

Fig. 2. SUB 4 and SUB 5 are located at the lines' interconnections.

message content to perceive the feeding breaker and its operational condition. An agent will not forward a message which arrived from the side of an open load switch. Moreover, an agent will not send a message to the side of an open load switch. This permits SSAs which own substations at lines' interconnections, to acquire information for alternative power sources (Fig. 2 SSAs of secondary substations with identification names SUB 4, SUB 5).

2) Data checking. Refers to the operation of evaluating the functional data of the substation (load switches' voltage, current, etc.). The objective is to realize a fault condition by confirming the total loss of voltage and current on all three phases of the substation's power supply. The completion of this behavior entails the completion of steady state and the transition to fault localization and isolation state.

Fault localization and isolation state. This behavior concerns the fault detection message exchange between adjacent SSAs. The agents acquire information regarding the operational conditions of their neighbors and compare the message contents with local substation data in order to localize the fault. The behavior consists of two subbehaviors.

1) Fault detection sender. Refers to the action of sending fault detection messages to neighbors. An SSA will not send a message to the side of an open load switch.

2) Fault detection receiver. Refers to the action of receiving fault detection messages. An SSA expects to receive the same number of messages with the number of messages it sent. Thereafter, the agent compares the fault detection part of the each message content with its local data. A nonidentical value means the occurrence of a fault at the side of the neighbor that sent the corresponding message. In this case, the agent isolates the fault from its side by opening the respective load switch and completes fault localization and isolation state.

Power restoration state. It consists of subbehaviors that the SSA initiates or completes depending on the position of local 
substation with respect to the fault, the messages which are received by the SSA, the reasoning upon local operational data, and the completion of other subbehaviors.

Breaker action sender. This subbehavior is initiated by the SSA that isolated the fault and which resides in a substation located at the side of the feeding breaker. It concerns the dispatching of a breaker action message toward the feeding breaker PSA.

Breaker action receiver-sender. This subbehavior is being initiated by the SSAs that reside in substations located along the above message propagation route.

Restoration request sender. It is a subbehaviour that is being initiated by the SSA that isolated the fault and which resides in a substation located at the opposite side of the feeding breaker. The subbehaviour concerns the dispatching of a restoration request message toward alternative power supplies direction.

Restoration request receiver-sender. This subbehavior is being initiated by the SSAs that reside in substations located along the restoration request message propagation route.

Restoration reply sender/receiver-sender. It is a subbehavior which is being initiated by an SSA that has acquired information for alternative power supplies (i.e., feeding breakers of medium-voltage lines other than its own). It concerns the dispatching of a restoration reply message toward the restoration requester SSA direction. This subbehavior concerns also the propagation of a restoration reply message achieved by the SSAs.

Confirmation sender/receiver-sender. This subbehavior is being initiated by an SSA that responded positively to a restoration reply message. It concerns the dispatching of a confirmation message toward the alternative power-supply direction. The positive response of an SSA mentioned before implies that the SSA has proceeded with a certain switching action. This action allows for the alternative power supply to restore power to the substations which precede the local substation position, toward the alternative power-supply direction. The SSA, which is the final receiver of the confirmation message, responds by closing the proper load switch of its substation.

The design and implementation of the restoration state behavior allow for the mutual completion of the incorporated subbehaviors. For example, the SSA may send a confirmation message inside the restoration reply sender/receiver subbehavior, if according to the information that the message includes, the SSA decides to proceed with a local switching action.

An SSA's behavior includes logical rules for deciding whether it will proceed with a switching action or pass the control over to an SSA which resides in the next substation. These rules allow for an SSA to control the power restoration of the substations in the segments of the MV line that covers the areas illustrated in Fig. 3.

The information that the SSA needs in order to initiate the rules mentioned before has been acquired during the fault localization and isolation procedure, as well as from the contents of incoming restoration reply messages.
TO ALTERNATIVE BREAKER

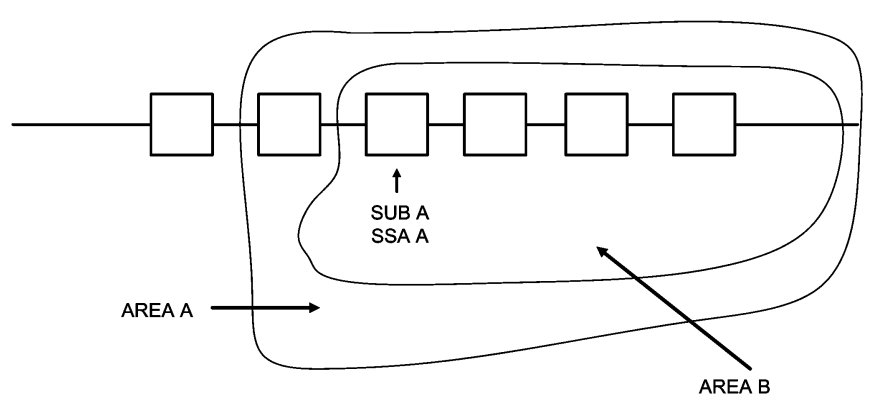

Fig. 3. Control areas of SSA A.

2) PSA: Generally, the behavior of a PSA concerns the dispatching of breaker information messages and the decoding of incoming breaker action messages. According to the content of a breaker action message, the PSA proceeds with operating the corresponding breaker in order to restore power to the segment of the line which extends from the breaker position to the substation that isolated the fault. Moreover, a PSA informs control engineers about the position of the fault.

Additional operations regarding the responses to incoming messages during the fault localization-isolation and power restoration procedure are also encapsulated in the PSA's general behavior body. These operations enable PSAs to participate in information exchange with the adjacent SSAs when appropriate, during the above procedures. The additional operations are structured in the same manner as the SSAs subbehaviors and concern the dispatching of fault detection messages, restoration reply messages, and responding to incoming confirmation messages.

\section{Communications}

The implementation of power-line communication (PLC) technology allows for the MV line to facilitate the communications among SSAs as well as between PSAs and SSAs. Extended information regarding the structure of the communication system as well as communication problems management is presented in [20].

\section{Cases Studied-Simulation Results}

The simplified MV distribution system of Fig. 2 has been simulated in order to draw certain conclusions concerning the implementation of the designed cooperative system. Fig. 4, illustrates a more detailed simulation topology.

The simulated Main Line is LINE 1. LINE 2 and LINE 3 are the alternative power sources for LINE 1. The breaker's identification name is BR $\mathrm{X}$ where $\mathrm{X}$ is the identification number of the corresponding line. The secondary substations identification names are SUB X, where $X$ is a number or string sign. The system's agents IDs derive from the respective secondary substations or breakers' identification names. For example, the SSA of substation SUB 1 is called SubAg 1 and the PSA of breaker BR1 is called BreakerAg1. The results below, derived from the agents' log files, have been created by the system's agents after the completion of restoration states for each case study. The 


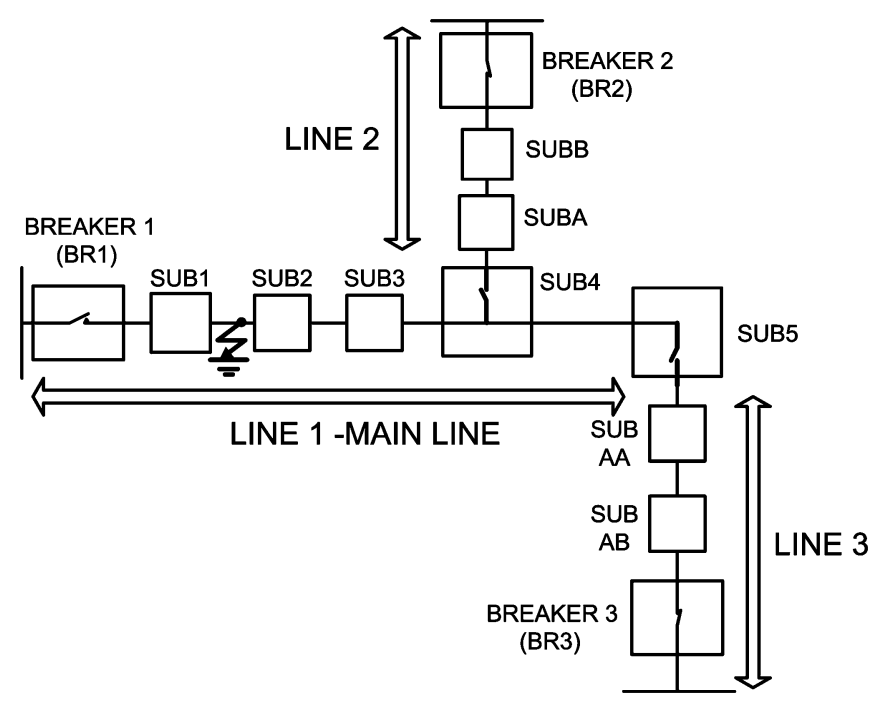

Fig. 4. Simulation topology.

PSAs' log files present the state of the breakers and the position of the fault formulated as: "Breaker BR X state is: $\mathrm{Y}$ and the position of the fault is $N$ from me" where $Y$ is "true/false" for "ON/OFF" and $N$ means that the fault is located and isolated between the $N$ th and the $(N$ th +1$)$ secondary substations; $N=1$ for substation SUB1.

The SSAs' log files include the time stamps as well as the states of the load switches after the completion of each operational state separately formulated as: "Switch $\mathrm{X}$ state is Y" where $\mathrm{X}$ is the identifying number of a load switch and $\mathrm{Y}$ is "true/false" for "ON/OFF." Especially after the detection of a fault condition, which is a case where steady state completes, the SSAs present in the $\log$ files are the states of the switches fault detectors formulated as "Switch X Fault Detector: $Z$," where $Z$ indicates whether the specific fault detector has detected fault current ( $Z=$ "true") or not ( $Z=$ "false"). Moreover, the $\log$ files include the breaker information that the SSAs acquired while in steady-state operation. The appearing time stamps vary, because the agents exhibit asynchronous behaviors. An explanation of the load switches' identification names is illustrated in Fig. 5. A typical collection of SSAs and PSAs log files (for Case Study 3) is shown in Appendix B.

\section{A. Case Study 1}

1) Premises:

- The secondary substations of LINE 1 are uniformly loaded. The load of each substation is $100 \mathrm{kVA}$.

- Fault occurred between SUB 1 and SUB 2.

- Breaker BR 2 excessive loading capabilities may serve the secondary substations from SUB 2 to SUB 5.

- Breaker BR 3 does not have any excessive loading capabilities.

2) Results: SSA SubAg 4 closed Switch 4 in order to serve the loads of substations SUB 2, SUB 3, SUB 4, and SUB 5 from the alternative breaker BR 2. SSAs SubAg1 and SubAg 2 isolated the fault by opening switches Switch 3 and Switch 2 of their local substation, respectively. PSA BreakerAg 1 closed

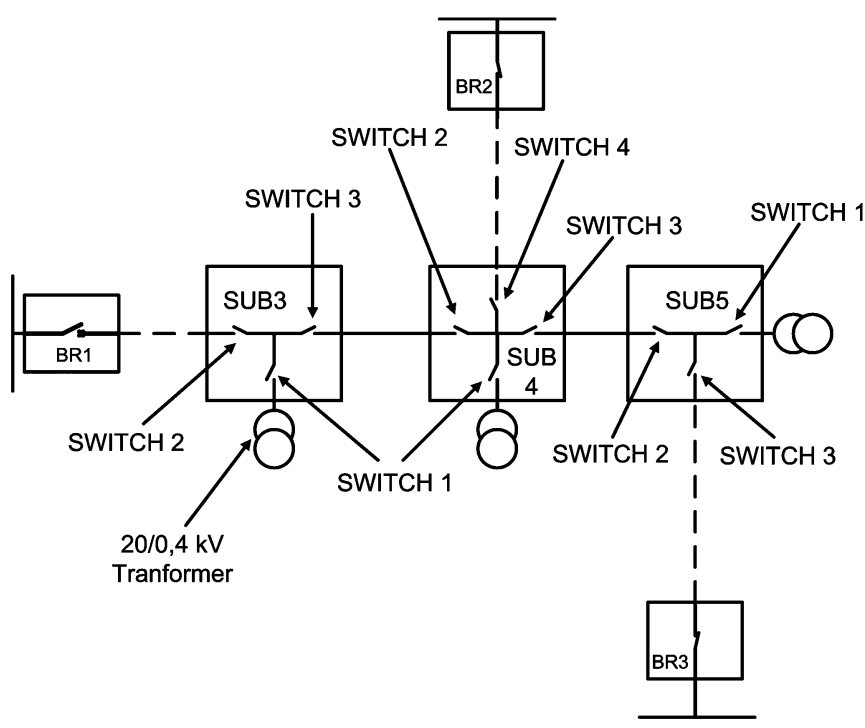

Fig. 5. Explanations of the load switches' identification names.

breaker BR 1 to safely supply substation SUB 1 . One-hundred percent of LINE 1 has been restored.

\section{B. Case Study 2}

1) Premises:

- The secondary substations of LINE 1 are not uniformly loaded. Substations SUB 1, SUB 3, and SUB 4 serve 100 $\mathrm{kVA}$. Substation SUB 2 serves $50 \mathrm{kVA}$ and substation SUB 5 serves $180 \mathrm{kVA}$ of load.

- Fault occurred between SUB 1 and SUB 2.

- Breakers BR 2 and BR 3 excessive loading capabilities may cumulatively restore power to substations SUB 2 to SUB 5.

2) Results: SSA SubAg 5 opened Switch 2 and closed Switch 3 in order to connect its substation to LINE 3 . Thereafter, SSA SubAg4 closed Switch 4 in order to serve loads of substations SUB 2 to SUB 4 from LINE 2. SSA's SubAg 5 switching actions preceded the ones of SubAg 4 in order to enable SubAg4 to decide that the excessive loading capabilities of breaker BR 2 suffice to restore power to substations SUB 2 to SUB 4. Onehundred percent of LINE 1 has been restored.

\section{Case Study 3}

1) Premises:

- The secondary substations of LINE 1 are not uniformly loaded. Substations SUB 1, SUB 2, SUB 3, and SUB 4 serve $100 \mathrm{kVA}$. Substation SUB 5 serves $180 \mathrm{kVA}$ of load.

- Fault occurred between SUB 1 and SUB 2.

- Breakers BR 2 and BR 3 excessive loading capabilities may not cumulatively restore power to substations SUB 2 to SUB 5.

2) Results: SSA SubAg 3 opened load switch 2 in order to isolate substation SUB 2 and reduce the loads to be restored. An analogous action was performed by SSA SubAg 5 by opening Switch 2 and closing Switch 3 in order to connect its substation to LINE 3. As a result, SSA SubAg 4 was enabled to close 
Switch 4 in order to connect the rest of the substations (SUB 3 and SUB 4) to LINE 2. Also, 82.7\% (480 kVA) of LINE 1 has been restored.

\section{CONCLUSION}

The authors designed and simulated a cooperative agent system that depends on situated and autonomous software agents with only local representation of the system's environment. The structure and operations of the agents' behaviors allow for the agents to exhibit totally asynchronous and uncoordinated functionality. Thus, a full distribution of decisions and actions has been achieved, which favors performance over fault management and power restoration procedures. As derived from the simulation results, the system's agents managed to exploit the maximum out of the alternative power sources in order to restore power to as many secondary substations as possible.

The implementation of the cooperative system architecture provides a desirable autonomy in terms of self-management to the power distribution network. The entire network becomes a living organism with self-healing capabilities. Finally, the power restoration procedures become more accurate and effective as well as less time consuming.

\section{APPENDIX A}

Agent. The frameworks' generic class which represents the agent entities inside an agent is agent identification (AID). AID defines the unique identification name of an agent entity.

Substation. It is a class that describes a secondary substation entity. Properties of a substation object are the load switches, the substation's load, the substation's identifier, the substation's type (local/neighbor), and the substation's position with respect to the fault.

Switch. It is a class which refers to a substation's load switch. Properties of a switch object are the load switch identifier, the load switch type (transformer switch or line switch), the load switch fault detector state, the load switch state, and, finally, the current, voltage, and power of the load switch.

Breaker. It is a class which describes a breaker entity. Properties of a breaker object are the breaker's identifier, type (feeding or alternative), load, excessive load capabilities, and state and breaker's fault detector state.

Power. It is a common class which refers to the power or load properties of other environment objects.

Fault position. It is a common class which refers to the relative position of a substation object with respect to the fault. Properties of a fault position object are the fault position order, by means of the number of substations which exist between the local substation and the fault, and the fault position type (primary/secondary). Fault position of type "primary" means that the fault is located at the side of the feeding breaker while "secondary" means the opposite.

Owns. It is a class which describes the "ownership" relation between an agent and a substation. Properties of an owns object are the owner agent and the substation owned.

Sees. It is a class which defines the location of a breaker with respect to the substation. Properties of a sees object are the load switch and the corresponding neighbor substation through which an agent "sees" a breaker.

Neighbors. It is a class which defines the "neighboring" relations among the substations. Properties of a neighbors object are the local substation, a substation or breaker adjacent to the local substation, and the load switch which is located at the side of the neighbor substation.

Breaker information. It is a class which describes the content of a message, where there is a PSA dispatch in order to inform the SSAs about a breaker's condition. Properties of a breaker information object are: the informer agent (PSA) and the information (breaker object).

Fault detection. It is a class which describes the content of the messages which the SSAs exchange in order to locate a line fault. Properties of a fault detection object are the agent (agent object) which sends the message and the substation, which the agent owns, object (substation object).

Breaker action. It is a class which describes the content of a message in which a SSA dispatches toward a PSA in order to inform about the position of an isolated fault and to order for a breaker operation. Properties of a breaker action object are the sender agent (AID object), the receiver agent (AID object), the breaker concerned (breaker object), and the operation ordered.

Restoration request. It is a class which describes the content of a message which an SSA dispatches in order to initiate a restoration procedure. Properties of a restoration request object are the sender agent (AID object), which is the agent that initializes the restoration procedure, the receiver agent (AID object), and the substation (substation object) owned by the sender agent.

Restoration reply. It is a class which describes the content of a message which is a reply to a restoration request message. Properties of a restoration reply object are the sender agent, the receiver agent, and the alternative power source object (breaker object).

Confirmation. It is a class which describes the content of a message which is dispatched from an SSA that proceeded with a switching action, toward an SSA that initially sent the restoration reply message. Properties of a confirmation object are the sender agent, the receiver agent, the alternative power source that is concerned (breaker object), and the confirmation value (usually an "OK" sign).

\section{APPENDIX B}

Agent's SubAg1 Log File

At time: 7:06:23 P.M. EET a fault condition detected; The information available for breakers is

breaker: BR1 is the feeding breaker with excessive loading capabilities: $500 \mathrm{kVA}$;

The load switches fault detectors' states are

Switch1 fault detector: false;

Switch2 fault detector: true;

Switch3 fault detector: true.

At time: 7:06:23 P.M. EET fault isolation finished

The load switches' states are:

Switch1 State: true;

Switch2 State: true;

Switch3 State: false. 
At time: 7:06:23 P.M. EET restoration process finished.

The load switches' states are

Switch1 State: true;

Switch2 State: true;

Switch3 State: false.

Agent's SubAg2 log file

At time: 7:06:18 P.M. EET a fault condition detected; The information available for breakers is

breaker: BR1 is the feeding breaker with excessive loading capabilities: $500 \mathrm{kVA}$;

The load switches Fault Detectors' states are

Switch1 fault detector: false;

Switch2 fault detector: false;

Switch3 Fault Detector: false.

At time: 7:06:23 P.M. EET fault isolation finished.

The load switches' states are

Switch1 State: true;

Switch2 State: false;

Switch3 State: true.

At time: 7:06:23 P.M. EET restoration process finished.

The load switches' states are

Switch1 State: true;

Switch2 State: false;

Switch3 State: true.

Agent's SubAg3 Log File

At time: 7:06:18 P.M. EET a fault condition detected.

The information available for breakers is

Breaker: BR1 is the feeding breaker with excessive loading capabilities: $500 \mathrm{kVA}$.

The load switches fault detectors' states are

Switch1 fault detector: false;

Switch2 fault detector: false;

Switch3 fault detector: false.

At time: 7:06:18 P.M. EET fault isolation finished.

The load switches' states are:

Switch1 state: true;

Switch2 state: true;

Switch 3 state: true

At time: 7:06:23 P.M. EET restoration process finished.

The load switches' states are

Switch1 sate: true;

Switch2 sate: false;

Switch3 sate: true.

Agent's SubAg4 Log File.

At time: 7:06:18 P.M. EET, fault condition detected.

The information available for breakers is

Breaker: BR1 is the feeding breaker with excessive loading capabilities: $500 \mathrm{kVA}$;

Breaker: BR2 is the 1st alternative breaker with excessive loading capabilities: $250 \mathrm{kVA}$.

The load switches Fault Detectors' states are:

Switch1 fault detector: false;

Switch2 fault detector: false;

Switch3 fault detector: false;

Switch4 fault detector: false.

At time 7:06:23 P.M. EET fault isolation finished.

The load switches' states are

Switch1 State: true;
Switch2 State: true;

Switch3 State: true;

Switch4 State: false.

At time: 7:06:23 P.M. EET restoration process finished.

The load switches' states are:

Switch1 sate: true;

Switch2 sate: true;

Switch3 sate: true;

Switch4 sate: true.

Agent's SubAg5 log file:

At time: 7:06:23 P.M. EET, a fault condition detected.

The information available for breakers is:

Breaker: BR1 is the feeding breaker with excessive loading capabilities: $500 \mathrm{kVA}$;

Breaker: BR3 is the 1st alternative breaker with excessive loading capabilities: $200 \mathrm{kVA}$.

The load switches fault detectors' states are:

Switch1 fault detector: false;

Switch2 fault detector: false;

Switch3 fault detector: false.

At time: 7:06:23 P.M. EET fault isolation finished.

The load switches's states are:

Switch1 state: true;

Switch2 state: true;

Switch3 state: false.

At time: 7:06:24 P.M. EET restoration process finished.

The load switches' states are:

Switch1 state: true;

Switch2 state: false;

Switch3 state: true.

Agent's BreakerAg1 log file:

breaker BR1 state is: true and the position of the fault is: 1 from me.

\section{REFERENCES}

[1] F. V. Gomes, S. Cameiro, J. L. R. Pereira, M. P. Vinagre, P. A. N. Garcia, and L. P. Araujo, "A new heuristic algorithm for large distribution systems," IEEE Trans. Power Syst, , vol. 20, no. 3, pp. 1373-1378, Aug. 2005.

[2] T. E. DcDermott, I. Drezga, and R. P. Broadwater, "A heuristic constructive method for distribution system reconfiguration," IEEE Trans. Power Syst., vol. 14, no. 2, pp. 478-483, May 1999.

[3] J. R. A. Rodriguez and A. Vargas, "Fuzzy-Heuristic methodology to estimate the load restoration time in MV networks," IEEE Trans. Power Syst., vol. 20, no. 2, pp. 1095-1102, May 2005.

[4] T. Nagata, H. Sasaki, and R. Yokoyama, "Power system restoration by joint usage of expert system and mathematical programming approach," IEEE Trans. Power Syst., vol. 10, no. 3, pp. 1473-1479, Aug. 1995.

[5] H.-J. Lee and Y.-M. Park, "A restoration aid expert system for distribution substations," IEEE Trans. Power Del., vol. 11, no. 4, pp. 1765-1770, Oct. 1996

[6] C.-C. Liu, S. J. Lee, and S. S. Venkata, "An expert system operational aid for restoration and loss reduction of distribution systems," IEEE Trans. Power Syst., vol. 3, no. 2, pp. 619-626, May 1988.

[7] Y.-Y. H. Lu, F.-C. Chien, Y. Liu, and J. P. J. Lin, "An expert-system for locating distribution system faults," IEEE Trans. Power Del., vol. 6, no. 1, pp. 366-372, Jan. 1991.

[8] T. Nagata and H. Sasaki, "A multi-Agent approach to power system restoration," IEEE Trans. Power Syst., vol. 17, no. 2, pp. 457-462, May 2002.

[9] T. Nagata, Y. Tao, H. Sasaki, and H. Fujita, "A multi-agent approach to distribution system restoration, circuits and systems," in Proc. 47th Midwest Symp., 2004, vol. 2, pp. II-333-II-336. 
[10] J. Yang, M. Montakhab, T. S. Davies, B. Carse, and A. G. Pipe, "Fault diagnosis of power distribution systems using a multi-agent approach," in Proc. UPEC Conf., Thessaloniki, Greece, 2003, pp. 629-632.

[11] M. Nordman and M. Lehtonen, "An agent concept for managing electrical distribution networks," IEEE Trans. Power Del., vol. 20, no. 2, pt. 1, pp. 696-703, Apr. 2005.

[12] InteliTEAM II S \& C Corporation [Online]. Available: www.sandc. com/products/Energyline/IntelliTEAM.

[13] T. Short, Electric Power Distribution Handbook. Boca Raton, FL: CRC, 2004.

[14] M. Amin, "Toward self-healing energy infrastructure systems," IEEE Comput. Appl. Power, vol. 14, no. 1, pp. 20-28, Jan. 2001.

[15] P. A. A. F. Wouters, P. C. J. M. vanderWielen, J. Veen, P. Wagenaars, and E. F. Steennis, "Effect of cable load impedance on coupling schemes for MV power line communication," IEEE Trans. Power Del., vol. 20, no. 2, pt. 1, pp. 638-645, Apr. 2005.

[16] J. Ferber, Multi-Agent Systems-An Introduction to Distributed Artificial Intelligence. London, U.K.: Addison-Wesley, 1999, pp. 8-13.

[17] JADE—Java Agent Development Framework (C) 2000 TILab S.p.A., (C) 2001 TILab S.p.A., (C) 2002 TILab S.p.A., (C) 2003 TILab S.p.A [Online]. Available: http://jade.tilab.com.

[18] G. R. Thomas, "Towards principles for the design of ontologies used for knowledge sharing, in formal ontology in conceptual analysis and knowledge representation," presented at the Int. Workshop on Formal Ontology Padova, Italy, Mar. 1993.

[19] FIPA ACL message structure specification, FIPA Spec. SC00061G 2002 [Online]. Available: http://www.fipa.org/specs/fipa00061/ SC00061G.html.

[20] I. S. Baxevanos and D. P. Labridis, "Implementing multi agent systems technology for power distribution network control and protection management," IEEE Trans. Power Del., vol. 22, no. 1, pp. 433-443, Jan. 2007.
Ioannis S. Baxevanos was born in Naoussa, Greece, on October 21, 1974. He received the Dipl.-Eng. and Ph.D. degrees from the Department of Electrical and Computer Engineering, Aristotle University of Thessaloniki, Thessaloniki, Greece, in 2001 and 2006, respectively.

Since 2001, he has been with the Power Systems Laboratory, Department of Electrical and Computer Engineering, Aristotle University of Thessaloniki. His research activities include the implementation of artificial-intelligence technology in the power systems field. His special interests are power distribution systems analysis, control and protection, with a special emphasis on power distribution systems automation.

Dimitris P. Labridis (S'88-M'90-SM'00) was born in Thessaloniki, Greece, on July 26, 1958. He received the Dipl.-Eng. and Ph.D. degrees from the Department of Electrical and Computer Engineering at the Aristotle University of Thessaloniki, Thessaloniki, in 1981 and 1989, respectively.

During 1982-2000, he was a Research Assistant, Lecturer, and Assistant Professor in the Department of Electrical and Computer Engineering at the Aristotle University of Thessaloniki. Since 2001, he has been an Associate Professor with the Department of Electrical and Computer Engineering at the Aristotle University of Thessaloniki. His research interests are power system analysis with a special emphasis on the simulation of transmission and distribution systems, electromagnetic- and thermal-field analysis, artificial-intelligence applications in power systems, power-line communications, and distributed energy resources. 